\title{
Analysis of electrical characteristics and magnetic field dependences of YBCO step edge and bicrystal grain boundary junctions for rf-SQUID applications
}

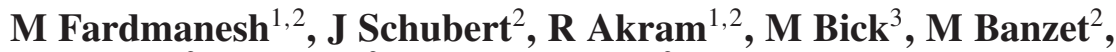 \\ W Zander ${ }^{2}$, Y Zhang ${ }^{2}$ and H-J Krause ${ }^{2}$ \\ ${ }^{1}$ Electrical and Electronics Engineering Department, Bilkent University, \\ Ankara 06800, Turkey \\ ${ }^{2}$ ISG, Research Center Juelich (FZJ), D52425 Juelich, Germany \\ ${ }^{3}$ CSIRO Telecommunication and Industrial Physics, Lindfield, NSW 2070, Australia
}

Received 19 November 2003, in final form 9 March 2004

Published 14 April 2004

Online at stacks.iop.org/SUST/17/S375

DOI: $10.1088 / 0953-2048 / 17 / 5 / 057$

\begin{abstract}
The dc characteristics and magnetic field dependences of $\mathrm{Y}-\mathrm{Ba}-\mathrm{Cu}-\mathrm{O}$ bicrystal grain boundary junctions (BGBJs) and step edge junctions (SEJs) were investigated for fabrication of rf-SQUIDs. Test junctions with up to $8 \mu \mathrm{m}$ widths as well as the junctions of the two types of junction-based rf-SQUID were studied. The SEJs typically showed lower $J_{\mathrm{c}}$ and higher $\rho_{\mathrm{N}}$ as compared to the BGBJs, resulting in close $I_{\mathrm{c}} R_{\mathrm{N}}$ products. All the BGBJs showed classical field dependent $I_{\mathrm{c}}$ following their junction width, resembling Fraunhofer patterns. The field sensitivity of the BGBJs' $I_{\mathrm{c}}$ led to low yield submicron BGBJ rf-SQUIDs partially impaired by the Earth's magnetic field. Two major behaviours of low and high field dependences of $I_{\mathrm{c}}$ were observed for the SEJs. Only the low field-sensitive SEJs resulted in micron size junction rf-SQUIDs not impaired by the Earth's magnetic field. The low field-sensitive SEJs led to low $1 / f$ noise magnetically stable rf-SQUIDs appropriate for applications in unshielded environments at $77 \mathrm{~K}$.
\end{abstract}

\section{Introduction}

Two widely used Josephson junction (JJ) types for fabrication of $\mathrm{Y}-\mathrm{Ba}-\mathrm{Cu}-\mathrm{O}$ rf-SQUIDs are the bicrystal grain boundary junctions (BGBJs) and the step edge junctions (SEJs) [1]. The properties of such JJs are strongly dependent on the detailed crystal structure at the grain boundary (GB) [1-3]. While the short coherence length in YBCO provides ease of obtaining the JJs by just a twinning in this material e.g. at a bicrystal substrate GB or at a step edge on a substrate, the control of the characteristics of the junctions is found to be difficult due to the need for high precision control of the growth at the GBs [1-3]. This is while the fabrication of rf-SQUIDs requires an almost precise critical current $\left(I_{\mathrm{c}}\right)$ controlled by the fabrication process $[4,5]$. This is due to the required optimum SQUID parameter, $\beta_{L}=2 \pi L I_{\mathrm{c}} / \Phi_{0} \cong 1$, where $L$ is the device inductance. The magnetic field dependences of the $I_{\mathrm{c}}$ of the junctions are also an important parameter for the rf-SQUIDs made for applications in unshielded environment [6]. There are advantages and disadvantages for both types of the BGBJ and SEJ technologies for making the rf-SQUIDs with respect to the fabrication process and designs as well as their dc characteristics and their $I_{\mathrm{c}}$ magnetic field dependences. These characteristics of the two technologies are studied in this work. Here we present the investigated $I-V$ characteristics and applied magnetic field $\left(B_{\mathrm{a}}\right)$ dependences of the $I_{\mathrm{c}}$ of both types of the junctions. This is to determine the more advantageous technique and find the optimum design parameters and limits imposed by each technology for fabrication of magnetically stable rf-SQUIDs [6]. Also the flux to voltage transfer function $\left(V_{\mathrm{s}-\mathrm{pp}}\right)$ modulation by the applied $B_{\mathrm{a}}$ as well as the characteristics of the junctions of the rf-SQUIDs made of both types of junctions, are investigated and presented here. 


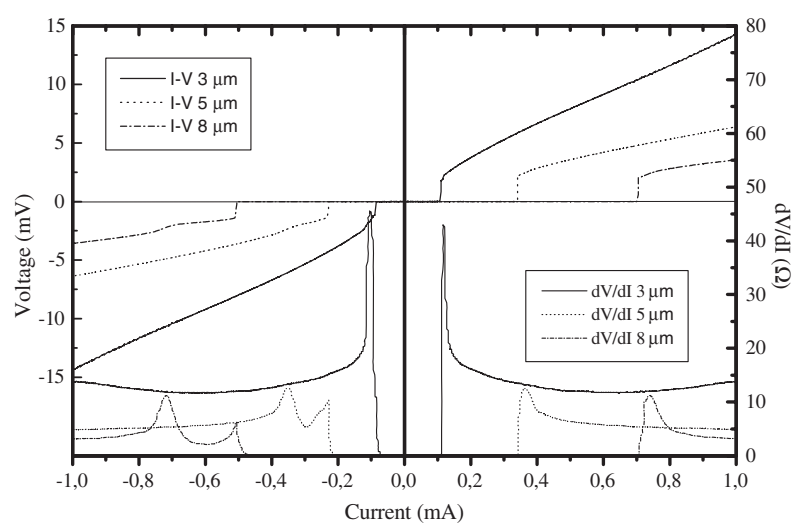

Figure 1. $I-V$ and the corresponding $\mathrm{d} V / \mathrm{d} I$ curves of 3,5 , and $8 \mu \mathrm{m}$ wide BGBJs on bicrystal $\mathrm{SrTiO}_{3}$ substrates at $7 \mathrm{~K}$.

The BGBJ and SEJ based arrays and rf-SQUID magnetometers and gradiometers were made of typically $200 \mathrm{~nm}$ thick YBCO film using pulsed laser deposition technique [7]. The bicrystal GB devices were made on symmetric $36.8^{\circ} \mathrm{GB}$ bicrystal $\mathrm{SrTiO}_{3}(100)$ substrates. The SEJ based samples were made on $\mathrm{LaAlO}_{3}(100)$ with steps prepared using an optimized combinatorial ion beam etching (CIBE) process, resulting in

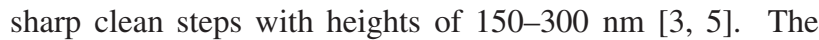
junctions were characterized by making contacts of gold wire bonds directly onto the surface of the films, resulting in contact resistances in the range of a few ohms at low temperatures. Junctions of the SEJ-SQUIDs were characterized by opening the SQUID washer areas while the designs of BGBJ rfSQUIDs used allowed this without destroying the devices [8]. Layout designs based on asymmetric multi-junction structures for BGBJ rf-SQUIDs were used to reduce the $1 / f$ noise of the devices [8-10]. Our BGBJ rf-SQUIDs were made with an about $0.8-1 \mu \mathrm{m}$ wide narrow junction, and $2-4 \mu \mathrm{m}$ wide dummy junctions. Further details on the designs of the SQUIDs are given in [8] and [9]. The SEJ rf-SQUIDs were made using our typical $3.5 \mathrm{~mm}$ diameter washer area designs with $100 \mu \mathrm{m}$ by $100 \mu \mathrm{m}$ loops and up to $5 \mu \mathrm{m}$ wide junctions [4-6]. The devices were characterized using either a liquid nitrogen Dewar based set-up with a three$\mu$-metal-layer shield, or a helium Dewar based system with a two- $\mu$-metal-layer shield and temperature stabilities better than $0.1 \mathrm{~K}$ [2]. Further details on the fabrication and characterization methodologies are given in [5] and [8].

\section{2. $I-V$ characteristics of the junctions}

Test junction characteristics were studied to find the limits imposed by each of the technologies and the optimum parameters in the rf-SQUID layout designs. Arrays of up to $8 \mu \mathrm{m}$ wide single BGBJs and SEJs were made to investigate the dc characteristics and magnetic field dependences on the junction width $\left(W_{\mathrm{j}}\right)$ of both types of junction. Also arrays of 5 $\mu \mathrm{m}$ wide 3-25 serial BGBJs were made in order to compare to the characteristics of SEJs with inevitable fourserial junctions forming at edges of the steps.

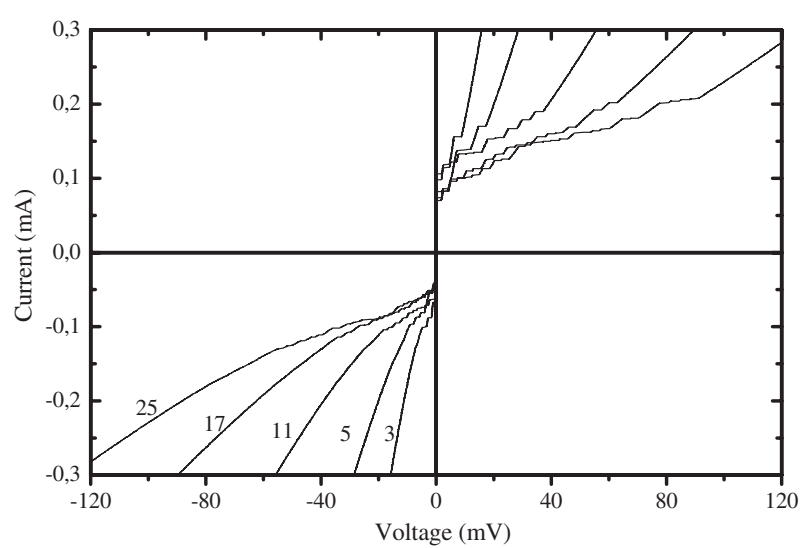

Figure 2. $I-V$ curves of $5 \mu \mathrm{m}$ wide $3-25$ serial BGBJ arrays on bicrystal $\mathrm{SrTiO}_{3}$ substrates at $5 \mathrm{~K}$.

\subsection{Junction parameters}

Critical current, $I_{c}$. The $I_{\mathrm{c}}$ of the BGBJs increased as the $W_{\mathrm{j}}$ increased but not proportionally, as shown for the $I-V$ characteristics of 3-8 $\mu \mathrm{m}$ wide BGBJs in figure 1 . The $I_{\mathrm{c}}$ ratios decreased further than the $W_{\mathrm{j}}$ ratios, which might be due to the side defects or slight non-uniformity of the barriers being more effective for smaller $W_{\mathrm{j}}$ [11]. This is also partly associated with the spread of the junction parameters as shown for the $5 \mu \mathrm{m}$ wide serial BGBJs in figure 2. The spread of $I_{\mathrm{c}}$ of our BGBJs is within the reported values $[11,12]$ and possibly caused by the optically observable defects at the substrate GBs [8]. While the $I_{c}$ of arrays of SEJs increased as the junction widths increased, a systematic dependence of $I_{\mathrm{c}}$ on $W_{\mathrm{j}}$ was not obtained and the spread of the junction parameters was higher than that of the BGBJs. The $I_{\mathrm{c}}$ of the SEJs was highly sensitive to the uniformity of the films and microstructure of the steps [3,5]. The SEJs made on the $150 \mathrm{~nm}$ deep ditches resulted in about one order of magnitude higher $I_{\mathrm{c}}$ values compared to that of the BGBJs with mostly flux flow characteristics. The effect of the step height and the film thickness have been previously reported [3]. Mostly SEJs made of quality $200 \mathrm{~nm}$ thick films on sharp steps with step heights above $200 \mathrm{~nm}$ showed resistively-capacitively shunted junction (RCSJ) characteristics where all our BGBJs showed RCSJ type behaviour. The BGBJ critical current densities $\left(J_{\mathrm{c}}\right)$ ranged within about $20-40 \mathrm{kA} \mathrm{cm}^{-2}$. The $J_{\mathrm{c}}$ of the SEJs typically was lower than that of the BGBJs, being more favourable for fabrication of rf-SQUIDs due to the need of very low $I_{\mathrm{c}}$ for obtaining an optimum SQUID parameter at $77 \mathrm{~K}$.

Normal resistance, $R_{N}$. The normal resistance, $R_{\mathrm{N}}$, of the junctions also scaled inversely with the $I_{\mathrm{c}}$ or $W \mathrm{~s}$, leading to similar $I_{\mathrm{c}} R_{\mathrm{N}}$ (or $J_{\mathrm{c}} \rho_{\mathrm{N}}$ ) values. The $R_{\mathrm{N}}$ of the 3,5 , and $8 \mu \mathrm{m}$ wide BGBJs in figure 1 are about 14,6 , and $2.5 \Omega$, respectively, giving sheet resistances $\left(\rho_{\mathrm{N}}\right)$ in the range of about $48-95 \mathrm{n} \Omega \mathrm{cm}^{2}$ and $I_{\mathrm{c}} R_{\mathrm{N}}$ values in the range of $1.8-2.1 \mathrm{mV}$ at $7 \mathrm{~K}$. The obtained $I_{\mathrm{c}} R_{\mathrm{N}}$ values are in the range of and slightly higher than the reported values for the BGBJs [11, 13-15]. The measured $R_{\mathrm{N}}$ of most of our BGBJs showed a slight temperature dependence, decreasing by about $5-10 \%$ as the temperature increased from $7 \mathrm{~K}$ to their $T_{\mathrm{c}}$. Relatively high $\rho_{\mathrm{N}}$ values were obtained for our SEJs [16]. The $R_{\mathrm{N}}$ of our 


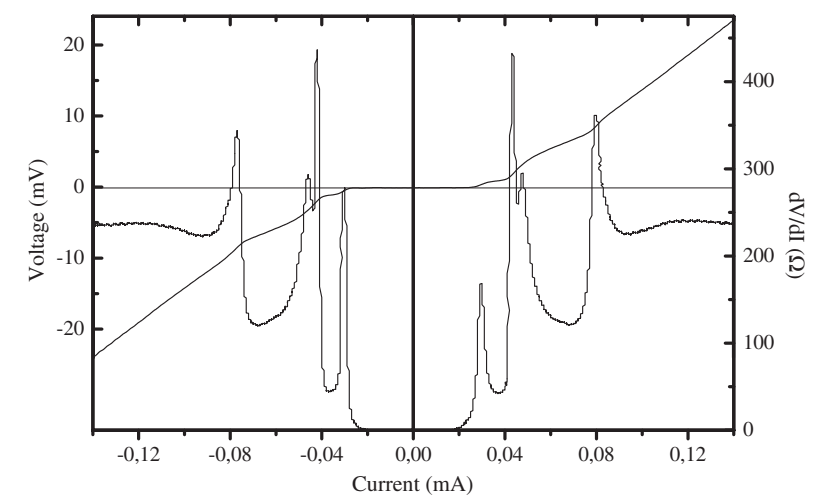

Figure 3. $I-V$ curve and the corresponding $\mathrm{d} V / \mathrm{d} I$ versus $I$ at $\sim 10 \mathrm{~K}$ of the $2 \mu \mathrm{m}$ wide SEJ of an rf-SQUID magnetometer made on $\mathrm{LaAlO}_{3}$ substrate with $255 \mathrm{~nm}$ deep steps.

SEJs was in the range of a few tens of ohms for $W_{\mathrm{j}}$ in the range of micrometres. Scaling of $R_{\mathrm{N}}$ and $I_{\mathrm{c}}$ of the SEJs with the $W_{\mathrm{j}}$ resulted in close $I_{\mathrm{c}} R_{\mathrm{N}}$ products in the range of $1 \mathrm{mV}$ at $T<10 \mathrm{~K}$ for various junction widths. The $I-V$ and $\mathrm{d} V / \mathrm{d} I$ curves of a $2 \mu \mathrm{m}$ wide junction on a $270 \mathrm{~nm}$ deep ditch are shown in figure 3 . While the $R_{\mathrm{N}}$ values of the RSJ-like behaviour SEJs mostly decreased with increase of temperature further than that of the BGBJs, the typical higher $\rho_{\mathrm{N}}$ of the SEJs is interpreted to be a major advantage of the SEJ technology in obtaining lower white noise devices [16].

Junction capacitance, $C_{j}$. The hysteretic under-damped (non-zero Steward-McCumber parameter, $\beta_{\mathrm{c}}=4 \pi e I_{\mathrm{c}}$ $\left.C R^{2} / h\right)$ behaviour of the junctions increased as the temperature decreased [17]. Cooling of the BGBJs resulted in $\beta_{\mathrm{c}}$ of about $2.3-2.5$ at $7 \mathrm{~K}$. The associated values of the $\beta_{\mathrm{c}}$ of the BGBJs led to junction capacitances $\left(C_{\mathrm{j}} \mathrm{s}\right)$ within the range of $5-8.5 \mu \mathrm{F} \mathrm{cm}^{-2}$ and about half typical reported values $[15,18]$. The associated $\beta_{\mathrm{c}}$ of the SEJs with hysteretic behaviour resulted in a junction capacitance in the range of 0.5 to a few $\mu \mathrm{F} \mathrm{cm}^{-2}$, well below the expected typical reported values for $\mathrm{Y}-\mathrm{Ba}-\mathrm{Cu}-\mathrm{O}$ GB JJs $[15,18,19]$. The relatively low capacitance and high $R_{\mathrm{N}}$ values of our SEJs suggest effective junction areas much smaller than the geometrical 'area' of the junctions $[6,16]$. The hysteretic $I-V$ characteristics could not be observed for our low $I_{\mathrm{c}}$ SEJs which is associated with the low ratio of Josephson coupling coefficient to the thermal fluctuations or simply high junction noise parameters, $\Gamma=2 \pi k_{\mathrm{B}} T / I_{\mathrm{c}} \phi_{0}$ [6]. The lower $I_{\mathrm{c}}$ and $C_{\mathrm{j}}$ of the SEJs are more favourable for the fabrication of the rf-SQUIDs [20].

\subsection{Nonlinearity}

The $I-V$ curves of our junctions exhibit a non-linear behaviour at $V \approx I_{\mathrm{c}} R_{\mathrm{N}}$ with deviation from the simple RSJ model for the larger $W_{\mathrm{j}}$ at low temperatures $[8,16]$. As shown in figure 1 , there is a strong non-linearity in the $I-V$ curve of the 5 and $8 \mu \mathrm{m}$ wide junctions as also reported for our SEJs [16]. This, which can enhance the noise of the devices, is associated with the Josephson flux motion effect occurring in junctions with widths larger than the Josephson penetration depth, $\lambda_{\mathrm{J}}=\left(h / 4 \pi e J_{\mathrm{c}} \mu_{0}(2 \lambda+d)\right)^{0.5}[17,21]$. The calculated $\lambda_{\mathrm{J}}$ of the BGBJS in figure 1 resulted in $W_{\mathrm{j}} / \lambda_{\mathrm{J}}$ of about 2,4 , and

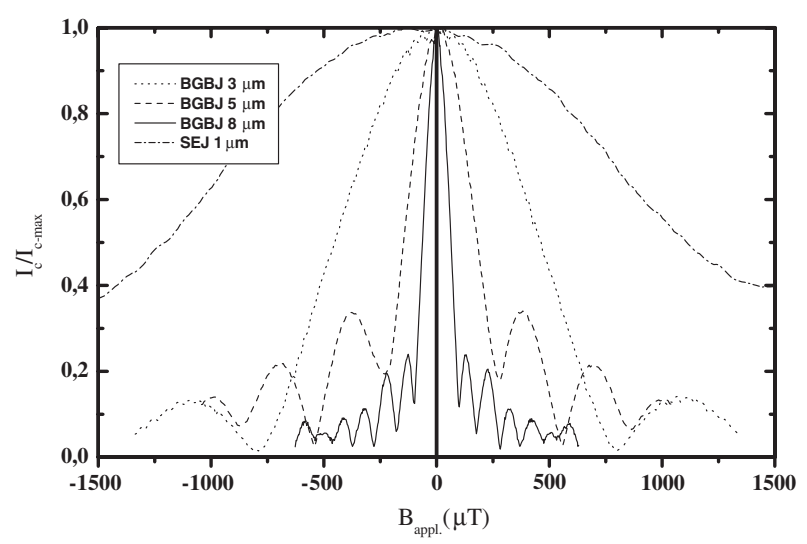

Figure 4. Magnetic field dependence of the normalized $I_{\mathrm{c}}$ of 3-8 $\mu \mathrm{m}$ wide BGBJs and a $1 \mu \mathrm{m}$ wide field-sensitive SEJ with $230 \mathrm{~nm}$ deep step at $T<10 \mathrm{~K}$.

7.8 for the 3,5 , and $8 \mu \mathrm{m}$ wide junctions respectively, which are within the range of the reported values $[15,21]$. Here we assume a uniform tunnelling current through a uniform barrier based on considering the junction widths not to be much larger than the $\lambda_{\mathrm{J}}$, especially for the SEJs due to their relatively low $J_{\mathrm{c}}$ [17]. As shown in the figure, the nonlinearity in the $I-V$ curves became prominent as the $W$ increased to $8 \mu \mathrm{m}$, resulting in $W_{\mathrm{j}} / \lambda_{\mathrm{J}}$ well above 4 [17]. The BGBJs in figure 1 showed clear linear $I-V$ curves as for short junction characteristics at temperatures higher than about 35,50 , and $70 \mathrm{~K}$ for the 3,5 , and $8 \mu \mathrm{m}$ wide junctions respectively, corresponding to $W_{\mathrm{j}} / \lambda_{\mathrm{J}}<\sim 2$. The same $W_{\mathrm{j}} / \lambda_{\mathrm{J}}$ ratio was also obtained as the criterion for the linear $I-V$ curves for our SEJs as the limiting factor for both types of junction in obtaining low noise devices [16]. The $I-V$ characteristics versus temperature of both junction types are presented elsewhere $[8,16]$. The $W_{\mathrm{j}} / \lambda_{\mathrm{J}}$ values for our SEJs were also obtained using the geometrical widths of the junctions. This rejects the possibility of having micro-short structures for the investigated SEJs in this study which might be concluded from their low $I_{\mathrm{c}}$ and $C_{\mathrm{j}}$ values. The above suggests an effective junction area proportional to ' $W_{\mathrm{j}}$ ' for both types of our junctions, as also confirmed by the dependence of the $I_{\mathrm{c}}$ on the junction widths.

\section{Magnetic field dependences of the junctions}

The applied magnetic field $\left(B_{\mathrm{a}}\right)$ dependence of the $I_{\mathrm{c}}$ of both types of junction was studied. The $I_{\mathrm{c}}$ versus $B_{\mathrm{a}}$ of all the BGBJs revealed a well defined Fraunhofer-patternlike behaviour scaled with the $I_{\mathrm{c}}$ of the junctions showing approximate proportionality to junction widths. The magnetic field dependences of the normalized $I_{\mathrm{c}}$ of various junction widths are shown in figure 4 . The sinc function type form of the field dependence of the $I_{\mathrm{c}}$ [8] and its deep modulations in figure 4 indicate an almost uniform current distribution through the areas of the junctions. While all the BGBJs showed classical $B_{\mathrm{a}}$ dependence scaling with their junction widths [16], a mixed relatively low and high $B_{\mathrm{a}}$ dependence of $I_{\mathrm{c}}$ was observed for our quality SEJs made on sharp CIBE steps [8]. Both types of low and high field-sensitive junction were made using $200 \mathrm{~nm}$ thick PLD YBCO films on $\mathrm{LaAlO}_{3}$ with 200-300 nm deep steps developed by an optimized CIBE 
process [22]. The CIBE steps were made using a low intensity $\left(\sim 0.1 \mathrm{~mA} \mathrm{~cm}^{-2}\right)$ and high energy $(500-600 \mathrm{eV})$ stationary $40^{\circ}$ angled ion beam along the step edges to obtain the approximate step height, and using a lower energy $(\sim 300 \mathrm{eV})$ rotating $45^{\circ}$ angled ion beam to get surface modified steps.

While the yield of the low field-sensitive devices has been improved by obtaining higher quality films on quality sharp steps through further optimization of the fabrication process [3], further investigation for controllability of the process is under investigation. The field sensitivity of our SEJs is found to be extremely sensitive to the fabrication process and highly dependent on the film and the steepness and surface of the steps. This is while the less steep shallow steps are found to mostly result in high field-sensitive junctions. A well defined $B_{\text {a }}$ dependence for an array of SEJs could not be obtained as they showed various field dependences as well as higher spread of the junction parameters compared to that of the BGBJs. The field dependence of a $1 \mu \mathrm{m}$ wide high field-sensitive junction is shown in figure $4 . I_{\mathrm{c}}$ of the SEJs with low field sensitivities showed very low $I_{\mathrm{c}}$ modulation (e.g. $\sim 25 \%$ for a $3 \mu \mathrm{m}$ wide junction [16]) versus $B_{\mathrm{a}}$ values up to about $1.5 \mathrm{mT}$, the limit of our characterization set-up [16]. The field dependence of all the junctions versus temperature was also investigated and the BGBJs and high $B_{\mathrm{a}}$ dependent SEJs showed similar sinc function characteristics with a slight change of the $\Delta B_{0}$, associated with the variations of the $\lambda[6,23]$. Typical $I-V$ behaviour of the investigated SEJs versus temperature is discussed in [24] and [8]. While the $I-V$ characteristics of our low field-sensitive SEJs versus temperature and magnetic field and in liquid nitrogen are discussed and presented in [24], [8], and [6] respectively, their systematic field dependences at temperatures close to $77 \mathrm{~K}$ could not be clearly investigated and presented so far. This has mainly been due to the low $I_{\mathrm{c}} \mathrm{S}$ and the relatively high noise parameter, $\Gamma$, of the SEJs at $T>60 \mathrm{~K}$ [6], as well as the sensitivity limits of the temperature variable characterization set-up. A systematic and detailed study of the $\lambda(T)$ dependence of the $\Delta B_{0}$ has not yet been obtained either due to the difficulty of fitting parameters at higher temperatures.

Our BGBJs showed $B_{\text {a }}$ dependence scaling with approximate $1 / W_{\mathrm{j}}^{2}$ ratio, the same as for the quality SEJs with high field sensitivities $[6,25,26]$. The measured dependence of the field period $\left(\Delta B_{0}\right)$ of the BGBJs and high field-sensitive SEJs versus $W_{\mathrm{j}}$ showed close $1 / W_{\mathrm{j}}^{2}$ dependence with the consideration of $\lambda \sim 180 \mathrm{~nm}$ at $T<10 \mathrm{~K}[6,25,26]$. While the $\Delta B_{0}$ of these junctions scaled closely with $1 / W_{j}^{2}$ ratio with a deviation of $4 \%$ at low temperatures, a $\Delta B_{0}=$ $6.1 \phi_{0} /\left(W_{\mathrm{j}}-\lambda\right)^{2}$ gave the best fit to our data for junctions widths above $\sim 3 \mu \mathrm{m}$. The $\Delta B_{0}=\alpha \phi_{0} / W_{\mathrm{j}}^{2}$ fitting approach to our junctions resulted in larger $\alpha$ for smaller junctions at low temperatures. The magnetic field dependence of $I_{\mathrm{c}}$ of the low field-sensitive SEJs could not be correlated to the $I_{\mathrm{c}}$ or the geometrical width of the junctions. This field dependence behaviour might be associated with the physical position of the low $I_{\mathrm{c}}$ junctions at the steps and/or their orientation with respect to the normal incident onto the substrate as well as the $B_{\mathrm{a}}$. This is while the junctions at the bottom of the steps, presumably shielded by the upper relatively thicker films at the edges of the steps [2], are considered to be the effective (lower $I_{\mathrm{c}}$ ) junctions among the four serial junctions resulting from the crossing microbridge across the ion beam etched ditch in the substrates [3]. The mixed $B_{\text {a }}$ dependences of the SEJs resulted in two distinct magnetic field behaviours of the SEJ rf-SQUIDs discussed in the following.

\section{4. rf-SQUID characteristics}

The dependences of both operating temperature range $\left(\Delta T_{\mathrm{op}}\right)$ and the magnetic field sensitivities of the flux-voltage transfer function $\left(V_{\mathrm{s}-\mathrm{pp}}\right)$ of the SQUIDs on the device junction types are investigated. The $\Delta T_{\mathrm{op}}$ and the $B_{\mathrm{a}}$ dependences of the $V_{\mathrm{s}-\mathrm{pp}}$ of the BGBJ and SEJ based SQUIDs are studied based on the dc characteristics and the $B_{\mathrm{a}}$ dependences of the $I_{\mathrm{c}}$ of the device junctions, discussed in the following.

\subsection{Operating temperature range of the SQUIDs}

The optimum working temperature of our BGBJ based rfSQUIDs varied from $\sim 20 \mathrm{~K}$ to about $T_{\mathrm{c}}$ of the films $[8,9]$. Based on the $I_{\mathrm{c}}$ versus temperature and the effective width of the BGBJs, an approximate optimum working temperature range close to $77 \mathrm{~K}$ was expected for our devices with $0.8-1 \mu \mathrm{m}$ wide operating junction in the designs [8]. This was based on optimum rf-SQUID parameter $\beta_{L}=2 \pi L I_{\mathrm{c}} / \Phi_{0} \cong 1$ for the layouts used and the expected $I_{\mathrm{c}}$ for the smaller junction of the SQUIDs. The observed spread and deviation of the optimum working temperature of our BGBJ rf-SQUIDs was mainly interpreted to be due to the spread of $I_{\mathrm{c}}$ values, as discussed for the BGBJ arrays in the earlier sections. This is while the arrays of junctions are physically very close to each other, compared to the junctions of an array of SQUIDs on one chip. The work for obtaining devices with more controlled parameters is in progress.

A detailed study of the dependence of the $V_{\mathrm{s}-\mathrm{pp}}$ of our SEJ based rf-SQUIDs with various junction widths on $\mathrm{LaAlO}_{3}$ with various step structures is presented elsewhere [6,11]. The dependence of the operating temperature range $\left(\Delta T_{\mathrm{op}}\right)$ of the SEJ rf-SQUIDs on the step structures and the SQUID junction widths was in good agreement with the $I_{\mathrm{C}}$ measurement of the junctions of the SQUIDs as well as the studied test junction arrays above [16]. Decrease of the junction width for the same step heights or increase of the step heights for similar junction widths reduced the $\Delta T_{\mathrm{op}}$ range of the devices, a detailed study of which has been previously reported $[5,7,16]$.

\subsection{Magnetic field dependences}

Based on the $B_{\mathrm{a}}$ dependence of the $I_{\mathrm{c}}$ of the BGBJs, a very low suppression of $V_{\mathrm{s}-\mathrm{pp}}$ of zero-field cooled BGBJ SQUIDs was expected under the Earth's magnetic field as also verified by the direct field sensitivity measurements of the devices. This was based on the consideration of the width of the narrow junction of the device and the estimated flux focusing factor effect due to the SQUID layouts [8]. The field dependence of the $V_{\mathrm{s}-\mathrm{pp}}$ of a BGBJ rf-SQUID with $1 \mu \mathrm{m} / 4 \mu \mathrm{m}$ asymmetric junction ratio is shown in figure 5. As also expected from the test junction characteristics, there was no major systematic suppression of the $V_{\mathrm{s}-\mathrm{pp}}$ by the fields well above the Earth's magnetic field. The symmetric notches at about $200 \mu \mathrm{T}$ are associated with the possible interference of the field dependence of the $I_{\mathrm{c}}$ of the 


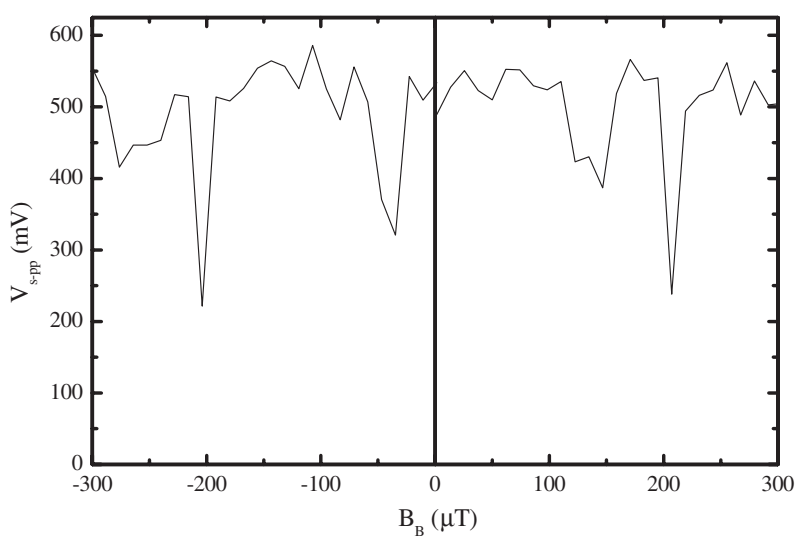

Figure 5. Magnetic field dependence of flux-voltage transfer function signal, $V_{\mathrm{s}-\mathrm{pp}}$, of a asymmetric junction bicrystal-GB rf-SQUID.

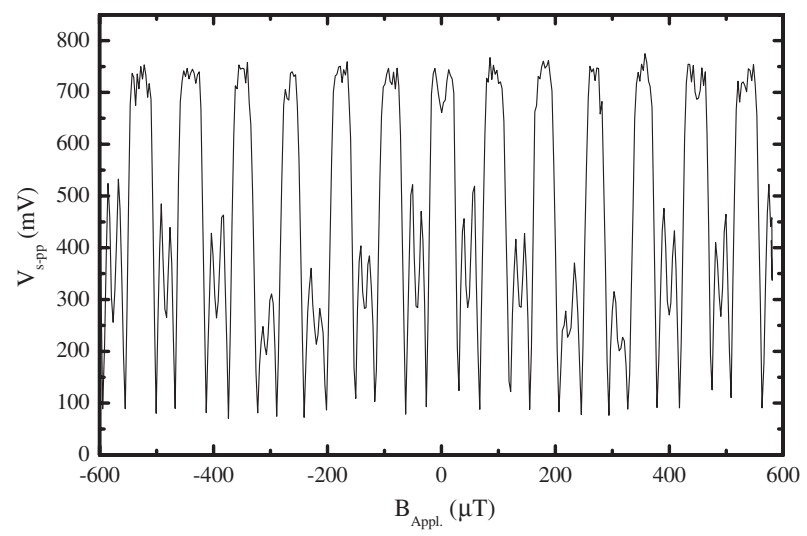

Figure 6. Normalized magnetic field dependence of $V_{\mathrm{s}-\mathrm{pp}}$ of a high field-sensitive $2 \mu \mathrm{m}$ wide SEJ rf-SQUIDs.

$4 \mu \mathrm{m}$ wide dummy junction of the device. The incompleteness of these notches is associated with a favourable possible slight non-uniformity of the $J_{\mathrm{c}}$ of the $4 \mu \mathrm{m}$ wide junction. The favourable non-uniformities might also be made intentionally, which is being further studied. The notches at the $-40 \mu \mathrm{T}$ and $150 \mu \mathrm{T}$ in figure 5 might be associated with the penetration of vortices close to the junctions [18] occurring at the larger magnetic fields during the cycling of the measurements.

While one classical type of $B_{\mathrm{a}}$ dependence was observed for our BGBJ rf-SQUIDs, two distinct relatively high and low $B_{\mathrm{a}}$ sensitivities were observed for the $V_{\mathrm{s}-\mathrm{pp}}$ of our SEJ rf-SQUIDs made on sharp CIBE steps. This was consistent with the field dependence for $I_{\mathrm{c}}$ of the isolated test junctions, showing two distinct $B_{\mathrm{a}}$ dependences. The field sensitivity of the test junctions was correlated to that of the SQUID junctions through the effective area of their patterns [9]. The field dependences of $V_{\mathrm{s}-\mathrm{pp}}$ of 2-3 $\mu \mathrm{m}$ wide junction rf-SQUIDs with high and low $B_{\mathrm{a}}$ sensitivities are shown in figures 6 and 7 respectively. The $B_{\mathrm{a}}$ dependence of the $I_{\mathrm{c}}$ of the SQUID junctions also followed that of the $V_{\mathrm{s}-\mathrm{pp}}$ of the SQUIDs $[8,16]$. As observed from figure 6, there is also a lower modulation of the $V_{\mathrm{s}-\mathrm{pp}}$, also indicating the existence of the effect of a low $B_{\mathrm{a}}$-sensitive junction in series. This modulation follows the trend with the $W_{\mathrm{j}}$ compared to that of the $3 \mu \mathrm{m}$ wide low $B_{\text {a }}$ sensitive SEJ device in figure 7. The multiple modulation types in figure 6 are interpreted to be due to the field dependent

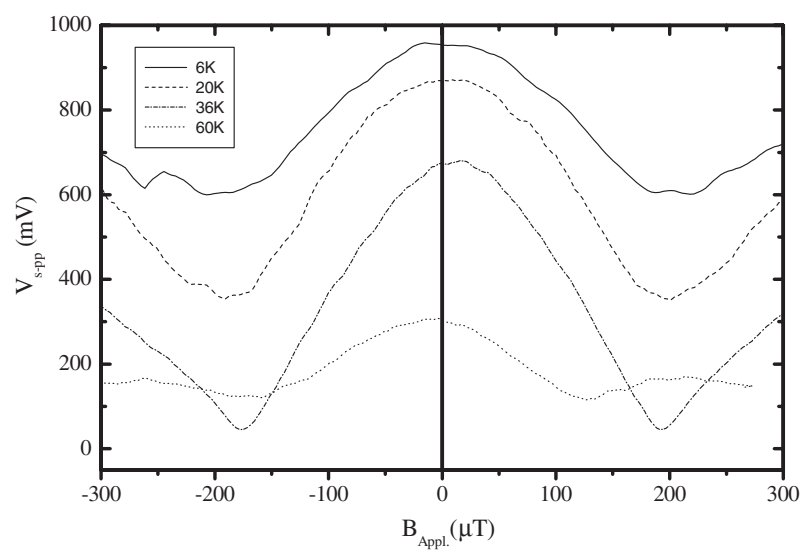

Figure 7. Magnetic field dependence of $V_{\mathrm{s}-\mathrm{pp}}$ versus temperature of a low field-sensitive SEJ rf-SQUID.

interferences in the $V_{\mathrm{s}-\mathrm{pp}}$ of the inevitable serial junctions in the SEJ rf-SQUIDs [1-3]. The study of the high $B_{\mathrm{a}}$ sensitivity of our SEJ rf-SQUIDs and its correlation with their junction parameters has been previously reported $[6,16]$.

The $V_{\mathrm{s}-\mathrm{pp}}$ of the low $B_{\mathrm{a}}$-sensitive devices dropped by less than about $10 \%$ under $B_{\mathrm{a}} \sim 50 \mu \mathrm{T}$, as also observed from figure 7. As for the SEJs, the temperature dependence of the modulation type of the $V_{\mathrm{s}-\mathrm{pp}}$ in figure 7 did not lead to any systematic temperature dependence associated with $\lambda_{L}(T)$ either. The field dependence of the $I_{\mathrm{c}}$ of the junctions of the SQUIDs with similar low $B_{\mathrm{a}}$ sensitivities also showed low $B_{\mathrm{a}}$ dependences in agreement with the field dependences of the corresponding SQUIDs [16]. The study of the drop of the $V_{\mathrm{s}-\mathrm{pp}}$ of high $B_{\mathrm{a}}$-sensitive devices led to the need for rf-SQUID layout designs with junction widths in the range of $0.6-1.2 \mu \mathrm{m}$ to obtain magnetically stable devices for applications in an unshielded environment $[6,16]$. This is while low $B_{\mathrm{a}}$-sensitive SEJs resulted in 2-3 $\mu \mathrm{m}$ wide junction magnetically stable rf-SQUIDs appropriate for operation under Earth's magnetic field. With the consideration of the typically lower $J_{\mathrm{c}}$ and higher $\rho_{\mathrm{N}}$ of the SEJs compared to that of the BGBJs, the low field-sensitive SEJs led to the preference of using SEJ technology for fabrication of the rf-SQUIDs for operation at $77 \mathrm{~K}$. However, a systematic investigation for obtaining a higher yield of low field-sensitive SEJs is further needed. The studied SEJ rf-SQUIDs in this work with lower $B_{\mathrm{a}}$ sensitivities also showed lower $1 / f$ noise levels compared to that of the higher $B_{\mathrm{a}}$-sensitive devices. Further investigation for correlating the noise characteristics and $B_{\mathrm{a}}$ dependence of the devices is also in progress.

\section{Summary and conclusions}

Junction arrays and rf-SQUIDs were made on both bicrystal $\mathrm{SrTiO}_{3}$ substrates, and $\mathrm{LaAlO}_{3}$ substrates with steps developed using a CIBE process. All the BGBJs showed RSJ type characteristics. This is while mostly SEJs with $\sim 200 \mathrm{~nm}$ deep steps and higher showed RSJ type characteristics. The SEJs had typically lower $J_{\mathrm{c}}$ and higher $\rho_{\mathrm{N}}$ values compared to those of the BGBJs, resulting in close $I_{\mathrm{c}} R_{\mathrm{N}}$ products. Comparison of the present $I-V$ characteristics of both our types of junction suggests that SEJs are more suitable junctions 
for obtaining lower white noise devices. All our characterized junctions showed clear linear $I-V$ curves as for short junction characteristics at temperatures corresponding to $W_{\mathrm{j}} / \lambda_{\mathrm{J}}<$ $\sim 2$ as a criterion for obtaining low noise devices in both technologies. This favourably resulted in linear flux flow free $I-V$ curves for both types of junction at $77 \mathrm{~K}$ with widths up to $8 \mu \mathrm{m}$, not imposing a limit for obtaining low noise micron size junction devices operating at liquid nitrogen temperature.

All the characterized BGBJs showed a well defined Fraunhofer-pattern-like magnetic field dependent $I_{\mathrm{c}}$, indicating an almost uniform junction barrier. A well defined $B_{\text {a }}$ dependence for an array of SEJs could not be obtained as they showed various field dependences as well as a higher spread of $I_{\mathrm{c}}$. The field sensitivity of the BGBJs led to the need for submicron-junction rf-SQUID designs, decreasing the yield of this kind of device with appropriate optimum operating temperature. While only one field dependence was observed for the BGBJs, two major relatively high and low field dependences were obtained for our SEJ devices. A relatively high field sensitivity of the $I_{\mathrm{c}}$ of our SEJs and the resulting SEJ rf-SQUIDs was correlated to the junction widths, as for the BGBJ devices. The high field-sensitive SEJs also resulted in a need for submicron junction widths for applications in an unshielded environment. Due to the low $J_{\mathrm{c}}$ of our $200 \mathrm{~nm}$ thick film quality SEJs on the CIBE steps, this resulted in rf-SQUID designs with high $B_{\mathrm{a}}$ sensitivities inappropriate for operation in an unshielded environment. The obtained SEJs with relatively low field-sensitive $I_{\mathrm{c}}$ resulted in rf-SQUIDs with low $B_{\mathrm{a}}$ sensitivities appropriate for operation under the Earth's magnetic field, while carrying junction widths of $2-3 \mu \mathrm{m}$. The low field sensitivity of some of our SEJs is associated with the structure of the low $I_{\mathrm{c}}$ junctions at the steps. Considering the effect of the $I-V$ characteristics of the junctions, the SEJs with low field-sensitive $I_{\mathrm{c}}$ are favourable in fabrication of low noise rf-SQUIDs for applications in an unshielded environment.

\section{References}

[1] Braginski A I 1996 SQUID Sensors: Fundamentals, Fabrication and Applications (NATO ASI Series) ed H Weinstock (Dordrecht: Kluwer-Academic) p 235

[2] Jia C L, Kabius B, Urban K, Hermann K, Schubert J, Zander W and Braginski A I 1992 The microstructure of epitaxial YBCO films on steep Stepps n LaAlO substrates Physica C 196 211-26

[3] Fardmanesh M, Schubert J, Banzet M, Zander W, Zhang Y and Krause J 2002 Physica C 372 240-5

[4] Zhang Y, Wolters N, Zeng X H, Schubert J, Zander W, Soltner H, Zi H R, Banzet M, Ruder F and Braginski A I 1998 Appl. Supercond. 6 385-90
[5] Fardmanesh M, Schubert J, Banzet M, Zander W, Zhang Y and Krause J 2001 Physica C 345 40-4

[6] Bick M, Schubert J, Fardmanesh M, Panaitov G, Banzet M, Zander W, Zhang Y and Krause H-J 2001 IEEE Trans. Appl. Supercond. 11 1339-42

[7] Schubert J, Siegert M, Fardmanesh M, Zander W, Proempers M, Buchal C, Lisoni J and Lei C H 2000 Appl. Surf. Sci. 168 208-14

[8] Fardmanesh M, Schubert J, Akram R, Bick M, Banzet M, Zander W, Zhang Y and Krause H-J 2003 IEEE Trans. Appl. Supercond. 13833

[9] Fardmanesh M, Schubert J, Akram R, Bick M, Zander W, Zhang Y, Banzet M and Krause J-H 2001 Asymmetric multi-junction YBCO rf-SQUID magnetometer and gradiometer designs on bi-crystal substrates and the noise and junctions characteristics ISEC: Proc. Int. Conf. on Superconductive Electronics (Osaka, Japan, 2001) unpublished

[10] Il'ichev E, Krivoy G S and Ijsselsteijn R P J 2002 Physica C 377 516-20

[11] Burkhardt H, Bruegman O, Rauther A, Schnell F and Schilling M 1999 IEEE Trans. Appl. Supercond. $93153-6$

[12] Enpuku K, Minotani T, Hiraishi F, Kandori A and Kawakami S 1999 IEEE Trans. Appl. Supercond. 9 3109-12

[13] Chaudhari P, Mannhart J, Dimos D, Tsuei D, Chi J, Oprysko M M and Scheuermann M 1988 Phys. Rev. Lett. $601653-6$

[14] Minotani T, Kawakami S, Kuroki Y and Enpuku K 1998 Japan. J. Appl. Phys. 237 L718-21

[15] Heinsohn J-K, Dittmann R, Rodriguez Contreras J, Scherbel J, Klushin A and Siegel M 2001 IEEE Trans. Appl. Supercond. 11 795-8

[16] Fardmanesh M, Schubert J, Akram R, Bozbey A, Bick M, Banzet M, Lomparski D, Zander W, Zhang Y and Krause H-J 2003 IEEE Trans. Appl. Supercond. 13873

[17] Barone A and Paterno G 1982 Physics and Applications of the Josephson Effect (New York: Wiley)

[18] Mitchell E E, Foley C P, Mueller K-H and Leslie K E 1999 Physica C 321 219-30

[19] Tarte E J, Wagner G A, Somekh R E, Baudenbacher F J, Berghuis P and Evetts J E 1997 IEEE Trans. Appl. Supercond. 7 3662-5

[20] Zhang Y 2001 Evolution of HTS rf-SQUIDs IEEE Trans. Appl. Supercond. 11 1038-42

[21] Shimakage H, Ono R H, Vale L R and Wang Z 2001 IEEE Trans. Appl. Supercond. 11 4032-5

[22] Fardmanesh M, Schubert J and Banzet M 1999 Combinatorial ion beam etching process for step edge junction rf-SQUIDs German Patent Specification 19954167 A1

[23] Vendik O G, Vendik I B and Kaparkov D I 1998 IEEE Trans. Microw. Theory Tech. 46 469-78

[24] Fardmanesh M, Schubert J, Akram R, Bick M, Zhang Y, Banzet M, Zander W and Krause H-J 2001 IEEE Trans. Appl. Supercond. 111363

[25] Rosenthal P A, Beasley M R, Char K, Colclough M S and Zaharchuk G 1991 Appl. Phys. Lett. 59 3482-4

[26] Dimos D, Chaudhari P and Mannhart J 1990 Phys. Rev. B 41 4038-49 